\title{
Coupling between Vias and the PCB Power-Bus
}

\section{G. Heinrich and S. Dickmann}

Faculty of Electrical Engineering, Helmut-Schmidt-Universität/Universität der Bundeswehr Hamburg, Germany

\begin{abstract}
In this paper, a method for the extraction of data for a through-hole vertical interconnect access (via) on a printed circuit board (PCB) is presented. It uses a matrix de-embedding algorithm and two-port theory to extract the elements of an assumed lumped via model. After that the influence of the via on the power-bus impedance and the coupling between the via and the power-bus are described using this model.
\end{abstract}

\section{Introduction}

In PCBs vias are used to provide electrical connections between different layers or between a signal component and the power-bus (PB). With respect to the electromagnetic compatibility (EMC) the coupling between the vias and the powerbus, through which they run, is of interest. Figure 1 shows a typical geometry for this problem including a via in a 4-layer PCB: line 1 above the power plane $(\mathrm{P})$ is connected with line 2 beneath the ground plane $(\mathrm{G})$ by a through-hole via.

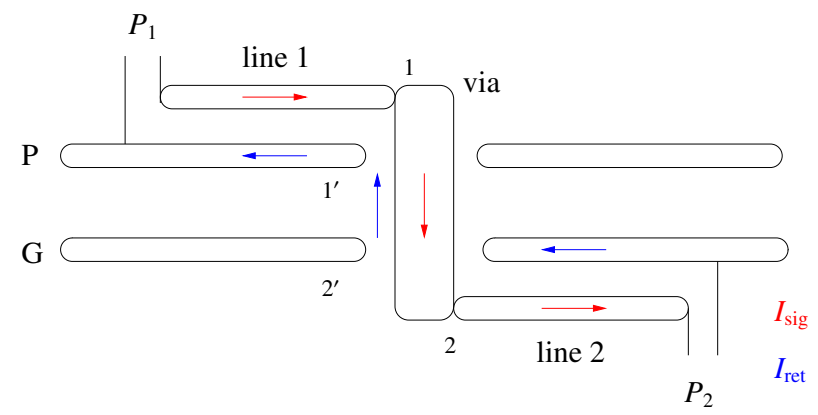

Fig. 1. Via through the power-bus connecting two lines.

The signal current $I_{\text {sig }}$ flows through line 1, the via and line 2. Its return current $I_{\text {ret }}$ flows on the PB planes beneath the

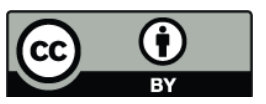

Correspondence to: G. Heinrich (gerd.heinrich@hsu-hamburg.de) lines and as displacement current through the PB adjacent to the via.

In Sect. 2 the derivation of an assumed lumped via model from the physics of the geometry (Fig. 1) and a model for the PB are decribed. After that two possible extraction procedures for the via model elements are presented in Sect. 3 . First, the $S$-parameters of a via are derived using a matrix de-embedding algorithm and afterwards two-port theory is used to extract the element impedances of an impedance via model. Second, the formerly extracted $S$-parameters are compared with the $S$-parameters calculated with a lumped via model.

In Sect. 4 the via model is finally used to describe the different interactions between the via and the PB: on the one hand the influence on the PB impedance and on the other hand the coupling between the via and the PB.

\section{Modelling of the via and the power-bus}

A via model is necessary to explain the effects of the via on the signal propagation between signal layers and also it is vital for the examination of possible interactions between the PB and the signal layers. Hence a suitable via model should correctly describe the following effects:

1. the electrical behavior of the via itself including parasitic effects,

2. the coupling mechanism between the via and the PB,

3. possible excitation sources for the $\mathrm{PB}$, and

4. the disturbances from the PB.

Because the dimensions of the via are small in comparison to the signal wavelength $\lambda$ up to several $\mathrm{GHz}$, the via model can be built up with lumped elements until at least $10 \mathrm{GHz}$. Here the via model is developed neglecting the transition discontinuity at the connection of the via and the lines.

Thus, the through-hole via in Fig. 1 can be modeled as shown in Fig. 2, where $Z_{\mathrm{PB}}$ is the $\mathrm{PB}$ impedance near the via

Published by Copernicus Publications on behalf of the URSI Landesausschuss in der Bundesrepublik Deutschland e.V. 


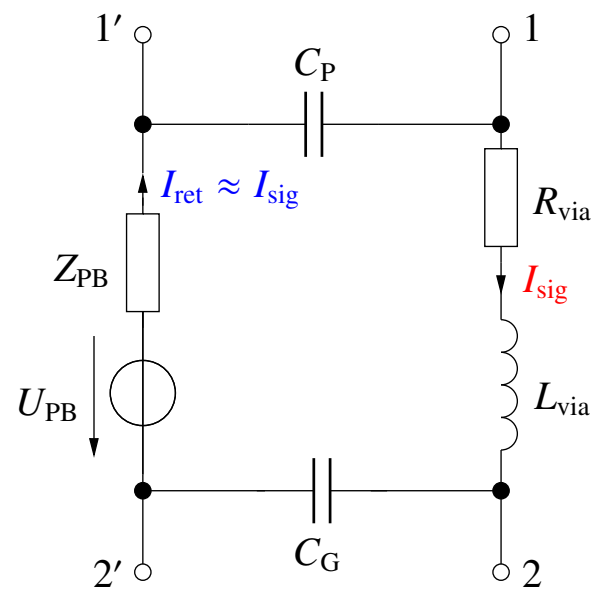

Fig. 2. Assumed lumped via model derived from the physics.

and $C_{\mathrm{P}}, C_{\mathrm{G}}$ are the coupling capacitances between the via and the PB planes. The via itself is described by an inductance $L_{\text {via }}$ in series with a resistance $R_{\text {via }}$, which accounts for the losses. Furthermore the voltage source $U_{\mathrm{PB}}$ models the induced voltage on the via due to the existing voltage distribution inside the $\mathrm{PB}$.

The impedance of the PB $Z_{P B}$ can be calculated approximately and analytically with a quite fast and accurate method. The modified modal summation (Hampe and Dickmann, 2005, 2007) is based on the cavity-model for an unpopulated rectangular $\mathrm{PB}$, which solves the 2-D-Helmholtz equation by using a series expansion of orthogonal modes (Okoshi, 1984), (Lei, 1999) and leads to a double summation expression for the impedance of a populated rectangular PB with dimensions $W_{x} \times W_{y}$ and thickness $W_{z}$ :

$$
\begin{array}{r}
Z_{i j}=\sum_{m=0}^{m_{\max }} \sum_{n=0}^{n_{\max }} \frac{c_{m n}\left(x_{i}, y_{i}\right) \cdot c_{m n}\left(x_{j}, y_{j}\right)}{Y_{m n}^{P}}, \\
Y_{m n}^{P}=Y_{m n}+\sum_{p=1}^{P} c_{m n}^{2}\left(x_{p}, y_{p}\right) \cdot Y_{P}^{*},
\end{array}
$$

with the source, observation and component port factors

$$
\begin{aligned}
c_{m n}\left(x_{i}, y_{i}\right)= & \cos \left(k_{x m} x_{i}\right) \cdot \operatorname{sinc}\left(k_{x m} \frac{P_{x_{i}}}{2}\right) \\
& \cdot \cos \left(k_{y n} y_{i}\right) \cdot \operatorname{sinc}\left(k_{y n} \frac{P_{y_{i}}}{2}\right),
\end{aligned}
$$

and the modal admittances

$$
Y_{m n}=G_{m n}+\mathrm{j} \omega C_{m n}+\frac{1}{\mathrm{j} \omega L_{m n}},
$$

whose elements $G_{m n}, C_{m n}$ and $L_{m n}$ account for the losses, the capacitive and inductive behavior of the different cavitymodes when they are in resonance. The modified impedances of the $P$ symmetrically connected lumped components are

$$
\begin{aligned}
Z_{P}^{*} & =\frac{1}{Y_{P}^{*}}=Z_{P}+\mathrm{j} \omega \Delta L_{i i}^{00}, \\
\triangle L_{i i}^{00} & =\sum_{m=0}^{m_{\max }} \sum_{n=0}^{n_{\max }} c_{m n}\left(x_{i}, y_{i}\right)^{2} \cdot L_{m n},
\end{aligned}
$$

where $\triangle L_{i i}^{00}$ accounts for the inductive behavior of all modes which are not in resonance and $k_{x m}=m \pi / W_{x}, k_{y n}=n \pi / W_{y}$, $C_{m, n}=1$ if $m, n=0$, and $C_{m, n}=2$ if $m, n \neq 0$. With the limits of the modal numbers:

$m_{\max }>2 W_{x} \sqrt{\varepsilon_{0} \varepsilon_{r} \mu_{0}} \cdot f_{\max }$

$n_{\max }>2 W_{y} \sqrt{\varepsilon_{0} \varepsilon_{r} \mu_{0}} \cdot f_{\max }$

all dominating waveguide modes are considered in the frequency range of interest, whereas the calculation effort is delimited.

\section{Extraction of the via model}

\subsection{De-Embedding algorithm}

The elements of the geometry in Fig. 1 form a cascade consisting of three parts: line 1, the through-hole via, and line 2 . In order to extract the $S$-parameters of the via, the influence of the lines must be de-embedded. This can be carried out with a matrix de-embedding using the scattering transfer parameters ( $T$-parameters) (Antonini, Scogna and Orlandi, 2003, 2004), (Heinrich and Dickmann, 2008) in the following steps:

1. numerical simulation or measurement of the entire geometry yields $S_{\text {geom }}, T_{\text {geom }}$,

2. simulation or measurement of the lines yields $T_{\mathrm{L} 1}, T_{\mathrm{L} 2}$ and

3. de-embedding of the lines yields:

$$
T_{\text {via }}=T_{\mathrm{L}_{1}}^{-1} \cdot T_{\text {geom }} \cdot T_{\mathrm{L}_{2}}^{-1},
$$

where the measurements are performed using a vector network analyzer (VNA) and the numerical simulations can be carried out with the FIT-based programme CST Microwave Studio (2009) (MWS). Finally the $S$-parameters of the via $S_{\text {via }}$ are calculated using (A1).

Another possiblity to characterize the lines is by calculating their $T$-parameters directly:

$$
\begin{aligned}
T_{\mathrm{L}} & =\frac{1}{a} \cdot\left(\begin{array}{cc}
\frac{a^{2}-b^{2}}{c} & b \\
-b & c
\end{array}\right), \\
a & =2 Z_{0} Z_{\mathrm{L}}, \\
b & =\left(Z_{\mathrm{L}}^{2}-Z_{0}^{2}\right) \sinh \left(\gamma L_{\text {line }}\right), \\
c & =2 Z_{0} Z_{\mathrm{L}} \cosh \left(\gamma L_{\text {line }}\right)+\left(Z_{\mathrm{L}}^{2}+Z_{0}^{2}\right) \sinh \left(\gamma L_{\text {line }}\right) .
\end{aligned}
$$



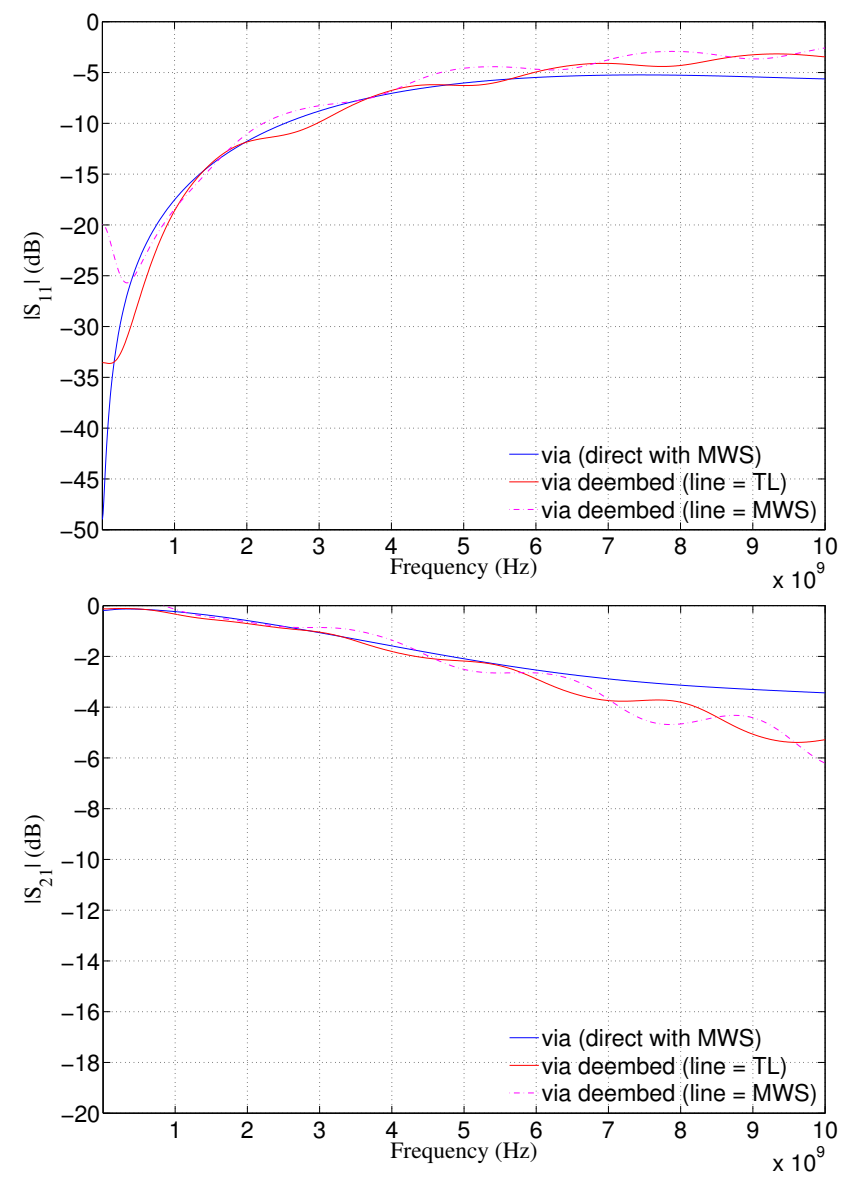

Fig. 3. $S$-parameters of a through-hole via: direct FIT simulation and extracted with the de-embedding of the lines.

from their characteristic impedance $Z_{\mathrm{L}}$ and propagation constant $\gamma$, which both depend on the geometry and the signal wavelength. They can be calculated with analytic formulas for the assumed quasi-TEM propagation on the microstrip lines in the frequency range of interest (Gupta, 1979), (Zinke, 2000).

When measuring the $S$-parameters of the geometry in Fig. 1, the microstrip lines are connected to the ports of the VNA via coaxial connectors. Therefore the connectors are attached to the lines and the PB plane next to them, which acts as the ground reference. After a "Full-2-Port-SOLT"calibration the reference planes for the measurements can be moved to the beginnings of the lines using the port extension option of the VNA with a time delay of approximately $37.6 \mathrm{ps}$ when using standard SMA-connectors. After performing this procedure only the influence of the lines has to be de-embedded from the measurement data, when parasitics of the connectors are neglected.

The $S$-parameters of a via (pad diameter $d_{\mathrm{pad}}=1.5 \mathrm{~mm}$, antipad diameter $d_{A P}=2 \mathrm{~mm}$ ), which connects two micro-
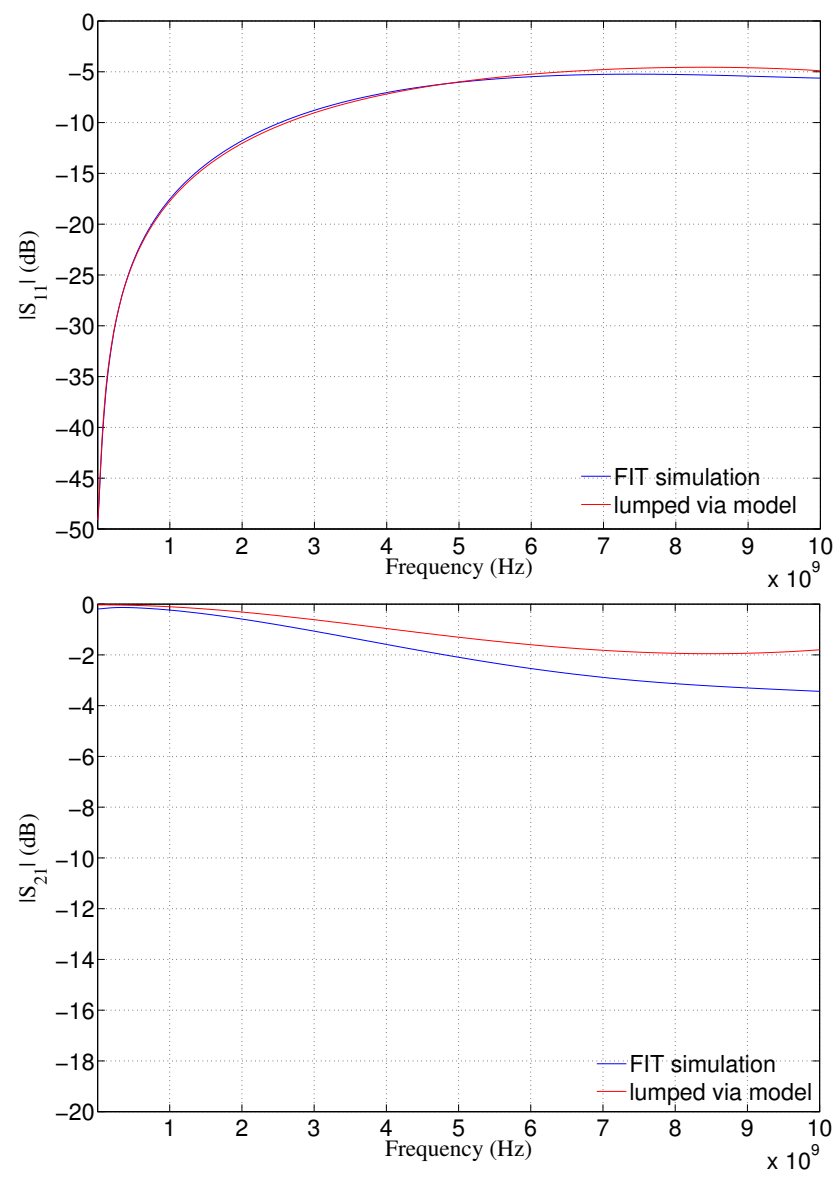

Fig. 4. $S$-parameters of a through-hole via: direct FIT simulation and results from the lumped via model.

strip lines (on layer 1 and $4, L_{\text {line }}=30 \mathrm{~mm}$ ), was extracted from the simulation results of the entire geometry using (5). Both lines have identical dimensions and were calculated with transmission line theory (TL) or simulated with MWS, respectively. Figure 3 shows a good coincidence between the results of the de-embedding and the direct simulation in MWS.

In Fig. 4 the $S$-parameters from the direct FIT-simulation is compared to the computation of the lumped via model (Fig. 2), where the following parameters were chosen: $R_{\text {via }}=0.35 \Omega, L_{\text {via }}=0.4 \mathrm{nH}$ and $C_{\mathrm{P}}=C_{\mathrm{G}}=0.5 \mathrm{pF}$. This shows that the assumed via model (Fig. 2) can be used to predict the $S$-parameters of the through-hole via in Fig. 1.

\subsection{Extraction of the via model elements}

For the investigation of the interactions between the signal layers and the PB, the elements of the assumed via models are required.

One possible way to get them, is by manual or automatic comparison of the $S$-parameters being extracted with the 


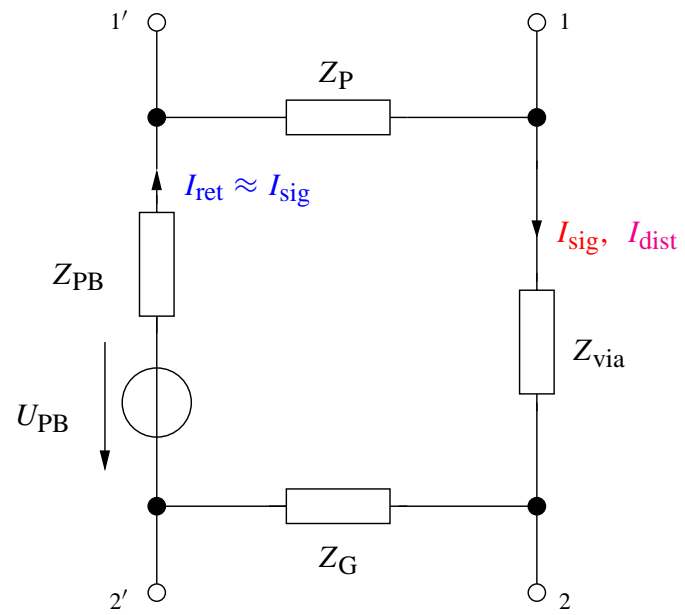

Fig. 5. Assumed impedance via model for extraction.

de-embedding algorithm and the results from the direct computation of a lumped via model like shown in Fig. 4. Thereby the model parameters are changed until both results match quite well.

Another possibility is the derivation of the via model elements directly from the result of the de-embedding algorithm. Therefore the extracted $T$-parameters of the via can be used to derive the elements of an impedance via model (Fig. 5), where the electrical behavior of the via itself is modelled with an impedance $Z_{\text {via }}$ and the coupling between the via and the $\mathrm{PB}$ planes are described with the coupling impedances $Z_{\mathrm{P}}$ and $Z_{\mathrm{G}}$.

After converting the $T$-parameters of the via from (5) into its impedance matrix with (A2) the elements of the assumed model for the through-hole via (Fig. 5) can be extracted considering symmetry $\left(Z_{11}=Z_{22}\right)$ and reciprocity $\left(Z_{21}=Z_{12}\right)$ (Antonini, Scogna and Orlandi, 2002), (Heinrich and Dickmann, 2008):

$$
\begin{gathered}
Z_{\mathrm{P}}=Z_{\mathrm{G}}=Z_{11}+Z_{12}, \\
Z_{\text {via }}=\frac{Z_{11}^{2}}{Z_{12}}-Z_{12}-Z_{\mathrm{PB}},
\end{gathered}
$$

where the input impedance of the PB at the location of the via $Z_{\mathrm{PB}}$ can be calculated with (1).

\section{Interactions between via and power-bus}

\subsection{Influence on the power-bus impedance}

To account for the influence of the via on the PB impedance, the extracted elements of the via models can be implemented in (1) in the same way as connected lumped components (2), (4):

$$
\begin{aligned}
& Y_{\mathrm{mn}}^{P}=Y_{\mathrm{mn}}+c_{\mathrm{mn}}^{2}\left(x_{\mathrm{via}}, y_{\mathrm{via}}\right) \cdot Y_{\mathrm{Via}}^{*}, \\
& Z_{\mathrm{via}}^{*}=Z_{\mathrm{via}}^{\mathrm{ges}}+j \omega \triangle L_{11}^{\mathrm{vv}}, \\
& Z_{\mathrm{via}}^{\mathrm{ges}}=Z_{\mathrm{via}}+Z_{\mathrm{P}}+Z_{\mathrm{G}},
\end{aligned}
$$

where the via impedances can be determined from (6) and (7). Also they can be calculated directly from the assumed parameters of the lumped via model in Fig. 2:

$$
\begin{aligned}
Z_{\mathrm{P}, \mathrm{G}} & =1 / \mathrm{j} \omega C_{\mathrm{P}, \mathrm{G}}, \\
Z_{\mathrm{via}} & =R_{\mathrm{via}}+\mathrm{j} \omega L_{\mathrm{via}} .
\end{aligned}
$$

\subsection{Coupling between via and power-bus}

For all injected signals the PB of a PCB acts like a multi-port. Because of the spreading waveguide modes inside the $\mathrm{PB}$, a disturbance injected at one location in the PB is transferred to other locations e.g. the connection locations of the signal components or the location of a via. Hence the induced voltages on the connection locations of these components $U_{\mathrm{SP}}$ can be calculated with:

$$
\left(\begin{array}{c}
U_{\mathrm{S} 1} \\
\vdots \\
U_{\mathrm{SP}}
\end{array}\right)=\underline{Z}_{\mathrm{PB}} \cdot\left(\begin{array}{c}
I_{\mathrm{PB}_{1}} \\
\vdots \\
I_{\mathrm{PB}_{\mathrm{N}}}
\end{array}\right),
$$

where $I_{\mathrm{PB}_{\mathrm{n}}}$ are currents injected on different locations between the parallel solid planes of the PB and the elements of the transfer impedance matrix $\underline{Z}_{\mathrm{PB}}$ are calculated with (1).

The signal return current $I_{\text {ret }}$, which flows as a displacement current through the dielectric of the PB near the via (Fig. 1), leads to the first coupling between the via and the PB: from the signal layers into the PB. It is a possible excitation source for new waveguide modes. Hence it causes a voltage disturbance on another location in the PB e.g. the connection of an IC:

$U_{\text {dist }}=Z_{12} \cdot I_{\text {ret }}$,

where $Z_{12}$ is the transfer impedance between the location of the via and the connection point.

Because of the capacitive coupling between the via and the $\mathrm{PB}$ planes there exists a coupling path from the PB into the signal layers. Consequently the voltage distribution inside the $\mathrm{PB}$ (voltage on the via $U_{\mathrm{PB}}$ ) couples into the signal layers. This causes a disturbance current $I_{\text {dist }}$ flowing through the via into the connected signal layers (Fig. 5), which may lead to signal integrity problems. 


\section{Conclusions}

In this paper, a through-hole via in a 4-layer PCB was examined. At the beginning, a lumped via model was derived from the physics. After that a matrix de-embedding algorithm for the extraction of the $S$-parameters of a via in a typical geometry was presented.

Then two different procedures for the extraction of the via model elements were shown. First, the elements of an assumed impedance via model can be derived directly from the $S$-parameters using two-port theory. Second, the $S$-parameters can be calculated with the assumed lumped via model. By means of a comparison with the previously extracted $S$-parameters suitable model parameters can be found.

Finally the derived via models were used to predict the influence of the via on the PB impedance and also to describe the coupling effects between the via and the PB.

\section{Appendix A Conversion formulas}

$$
\begin{aligned}
& S_{\mathrm{via}}=\frac{1}{T_{\mathrm{via} 22}} \cdot\left(\begin{array}{rl}
T_{\mathrm{via} 12} & \operatorname{det}\left(T_{\mathrm{via}}\right) \\
1 & -T_{\mathrm{via} 21}
\end{array}\right) . \\
& Z=\frac{Z_{0}}{a_{1}-a_{2}} \cdot\left(\begin{array}{cc}
-\left(a_{1}+a_{2}\right) & b_{2} a_{1}-b_{1} a_{2} \\
-2 & b_{2}-b_{1}
\end{array}\right), \\
& a_{1}=T_{11}+T_{12}, \quad a_{2}=T_{21}+T_{22}, \\
& b_{1}=T_{11}-T_{12}, \quad b_{2}=T_{21}-T_{22} .
\end{aligned}
$$

\section{References}

Hampe, M. and Dickmann, S.: Damping of Cavity-Mode Resonances in PCB Power-Bus Structures Using Discrete Capacitors, IEEE T. Electromagn. C., 47, 880-880, 2005.

Hampe, M. and Dickmann, S.: Single Summation Expression for the Impedance of Rectangular PCB Power-Bus Structures Loaded With Multiple Lumped Elements, IEEE T. Electromagn. C., 49, 58-67, 2007.

Heinrich, G. and Dickmann, S.: On the Influence of Vias on the PCB Power-Bus, Proceedings of the International Symposium on EMC (EMC Europe 2008), Hamburg, 85-90, 2008

Lei, G.-T., Techentin, R. W., and Gilbert, B. K.: HighFrequency Characterization of Power/Ground-Plane Structures, IEEE Transactions on Microwave Theory and Techniques, 47, 562-569, 1999.

Antonini, G., Scogna, A. C., and Orlandi, A.: Equivalent Network Synthesis for Via Holes Discontinuities, IEEE Transactions on Advanced Packaging, 25, 528-536, 2002.

Antonini, G., Scogna, A.C., and Orlandi, A.: S-Parameters Characterization of Through, Blind, and Buried Via Holes, IEEE Transactions on Mobile Computing, 2, 174-184, 2003.

Antonini, G., Scogna, A. C., and Orlandi, A.: De-Embedding Procedure Based on Computed/Measured Data Set for PCB Structures Characterization, IEEE Transactions on Advanced Packaging, 27, 597-602, 2004.

Okoshi, T.: Planar Circuits for Microwaves and Lightwaves, 1984.

Gupta, K. C., Garg, R., and Bahl, I. J.: Microstrip lines and slotlines, Artech House Microwave Library, 87-90, 1979.

Zinke, O. and Brunswig, H.: Hochfrequenztechnik I - Hochfrequenzfilter, Leistungen, Antennen, 6. Auflage, Springer-Verlag, 164-168, 2000.

CST Microwave Studio: http://www.cst.com/Content/Products/ MWS/Overview.aspx, 2009. 